Int. J. Dev. Biol. 58: 363-368 (2014)

doi: $10.1387 / \mathrm{ijdb} .140029 \mathrm{mu}$

\title{
Differential expression of arid5b isoforms in Xenopus laevis pronephros
}

\author{
RONAN LE BOUFFANT, ANNE-CLAIRE CUNIN, ISABELLE BUISSON, JÉRÔME CARTRY, \\ JEAN-FRANÇOIS RIOU and MURIEL UMBHAUER* \\ Sorbonne Universités, UPMC Univ Paris 06 and CNRS, UMR7622 Developmental Biology, Paris, France
}

\begin{abstract}
Arid5b belongs to the ARID family of transcription factors characterised by a helixturn-helix motif- based DNA-binding domain called ARID (A-T Rich Interaction Domain). In human, alternative splicing leads to long and short isoforms (isoform1 and 2, respectively) which differ in their N-terminal part. In this study, we report the cloning and expression pattern of Xenopus laevis arid5b. We have isolated a full length cDNA that shows homology with the human arid5b isoform 1. Furthermore, 5'RACE experiments revealed the presence of a shorter isoform equivalent to the human isoform2. Temporal expression analysis by RT-qPCR indicated that $X$. laevis arid5b isoform 1 and isoform2 are differentially expressed during development. Isoform 1 is strongly expressed maternally, while isoform 2 expression is essentially restricted to tailbud stages. Spatial expression analysis by whole mount in situ showed that arid5b is predominantly expressed in the developing pronephros. Arid5b mRNAs are detected in the antero-dorsal part of the pronephros anlage at the early tailbud stage and later on, in the proximal part of the pronephric tubule. RT-qPCR analyses with primers that allow to discriminate isoform 1 from isoform 2 showed that the latter is enriched in the pronephros anlage. In agreement with a specific pronephric signature of the isoform2, we also observed that isoform 2 but not isoform 1 is upregulated in animal caps induced to form pronephric tissue in response to activin $A$ and retinoic acid. These results indicate that the two arid5b isoforms are differentially expressed and likely play different roles during early Xenopus development.
\end{abstract}

KEY WORDS: arid5b, Xenopus, pronephros

The ARID (A-TRich Interaction Domain) is a helix-turn-helix motifbased DNA-binding domain, conserved in eukaryotes, that defines the ARID family of transcription factors. The human ARID family can be divided into seven subfamilies (ARID1, ARID2, ARID3, ARID4, ARID5, JARID1 and JARID2) based both on degree of homology within the ARID domain, as well as similarity between highly variable non-ARID domain structures. The founding members, murine Bright (ARID3A) and Drosophila dead ringer (Dri), were independently cloned on the basis of their ability to selectively bind to AT-rich DNA sequences but this behaviour is not an intrinsic feature of the ARID domain since the majority of ARID subfamilies bind DNA without obvious sequence preference (Patsialou et al., 2005). ARID-encoding genes are involved in a variety of biological processes including regulation of cell cycle, gene expression, differentiation, embryonic development, transcriptional regulation and chromatin-remodeling. The ARID protein Osa has been shown to associate with the SWI/SNF complex in both Drosophila and humans, suggesting that the function of ARID proteins in chromatin remodelling has been conserved through evolution (Collins et al., 1999, Kozmik et al., 2001).

Arid5b, also called Desrt or MRF-2 (modulator recognition factor-2), was originally cloned thanks to its ability to bind sequences in the transcriptional modulator of the human cytomegalovirus major immediate- early promoter (Lubon et al., 1989). Its ARID domain, whose three-dimensional structure has been solved, binds preferentially to an AT-rich core sequence (Whitson et al., 1999). Arid5b has been found to associate with the jmjC demethylase PHF2. Assembly of the PHF2-Arid5b complex, its recruitment to target promoters, and its $\mathrm{H} 3 \mathrm{H} 9 \mathrm{Me} 2$ demethylase activity are

Abbreviations used in this paper: aa, amino acid; ARID, A-T rich interaction domain; nt, nucleotide; RA, retinoic acid.

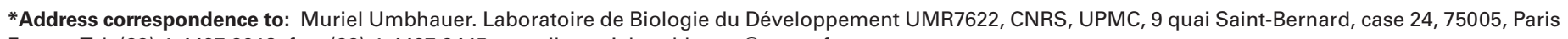
France.Tel: (33)-1-4427-3918: fax: (33)-1-4427-3445. e-mail: muriel.umbhauer@upmc.fr
}

Supplementary Material (3 figures and 2 tables) for this paper is available at: http://dx.doi.org/10.1387/ijdb.140029mu 
dependent on protein kinase A activity (Baba et al., 2011). During mouse organogenesis, arid5b displays a complex and highly dynamic pattern of expression. It is first expressed in the intermediate mesoderm and subsequently in the nephrogenic cords of the urogenital ridges. Arid5b is also detected in the limbs, the myotomes, the oro-naso- pharyngeal ectoderm and the underlying mesenchyme, the otic vesicles, the gut and its derivatives, and transiently in the liver. Arid5b mutant mice generated by gene targeting have reduced viability, pronounced growth retardation, and a high incidence of abnormalities of the reproductive organs (Lahoud et al., 2001). They also show significant reductions in lipid accumulation and weight gain in postnatal and adult life (Whitson et al., 2003). Arid5b is required for adipogenesis and to maintain normal functions in mature adipocytes. Knockdown of Arid5b in mature 3T3-L1-derived adipocytes activates both lipolysis and triglyceride synthesis, and causes a significant increase in the ratio of glycerol release to free fatty acid release (Yamakawa et al., 2010, Yamakawa et al., 2008). Arid5b is highly expressed in the cardiovascular system and is believed to play essential roles in smooth muscle cell differentiation and proliferation (Watanabe et al., 2002). In homozygous arid5b mutant mice, kidneys are small showing often degraded glomeruli with defects in smooth muscle cell number and location. Skeletal abnormalities, including defects in the patterning of the ribs and sternum, have also been described (Schmahl et al., 2007).

Although the temporal and spatial pattern of expression of arid5b during embryogenesis have been described in mouse (Ristevski et al., 2001), there is no detailed expression data available for non mammalian vertebrates. We report the cloning of two arid5b isoforms in Xenopus laevis and describe their expression patterns during development.

\section{Results and Discussion}

\section{Molecular cloning of $\mathrm{X}$. laevis arid5b}

In order to clone a full coding sequence of arid5b in Xenopus, we started from a partial IMAGE clone sequence (no 686,6480), and obtained the missing 5' sequence by RACE PCR. A 3570 nucleotides ( $\mathrm{nt}$ ) clone was amplified by end-to-end PCR (GenBank accession no. HG518326). Sequence analysis revealed an open reading frame encoding a predicted 1187 amino acids (aa) protein (Fig. S1). This protein displays $87.6 \%$ identity with a predicted $X$. tropicalis protein sequence deduced from gene models (accession no. XM_002939542), 57.5\% identity (82.2\% similarity) with the long human Arid5b isoform 1 , and $56.7 \%$ identity (80.7\% similarity) with the murine long isoform $\alpha$. The ARID domain (aa 324-418) is highly conserved, with more than $90 \%$ identity observed with other vertebrate Arid5b sequences (Fig.1). The conserved sequence includes a lysine residue (lys-342) at a position homologous to the lysine of the long murine isoform $\alpha$ (lys-336) whose demethylation by PHF2 promotes recruitment of PHF2-Arid5b complex to promoters (Baba et al., 2011). Blast analysis on the $X$. lævis genome 6.0 scaffolds shows that the long isoform is encoded by two genes located on scaffolds 9729 and 48311, respectively, which probably represent pseudoalleles resulting from $X$. lævis allotetraploidy. In a similar way to human and murine arid5b, the long $X$. lævis isoform is encoded by ten different exons (table S1,S2).

We further investigated whether a shorter isoform homologous to the short human arid5b isoform was also detectable. Human arid5b isoform 2 is generated by alternative splicing resulting in the replacement of phe-244 by a start methionine. Using 5' RACEPCR, we were able to clone a partial $X$. lævis arid5b sequence of 368nt containing an ORF encoding a 91 aa polypeptide where phe-244 is replaced by a methionine. The following aa are identical to those of the long isoform (ala-245-leu-334). The $95 \mathrm{nt}$ sequence located upstream to this ATG codon does not contain any other in frame ATG codon, but three stop codons indicating that it probably encodes the 5'UTR of a shorter isoform mRNA. Blast analysis on the $X$. lævis genome revealed that this sequence is encoded by a novel exon (exon 4b, table S1,S2), while the ala-245-leu-334 sequence is encoded by exons 5 and 6 , supporting the idea of an isoform generated by alternative splicing. Nested 3'RACE-PCR was carried out to clone the full sequence encoding this putative short isoform. The first primer was located $61 \mathrm{nt}$ upstream of the putative start ATG, and the nested primer 1307nt downstream of this ATG. A 2163nt sequence was cloned containing 1529nt of
A
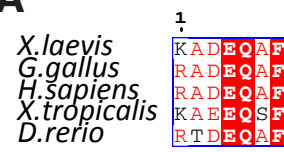

10

20

30

40

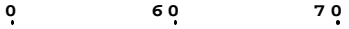

$\mathrm{ET} I$
$\mathrm{FT}$ X.laeyis G.gallus X.tropicalis D.rerio

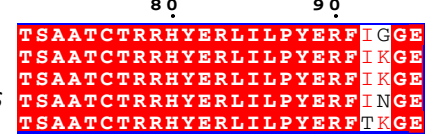

B

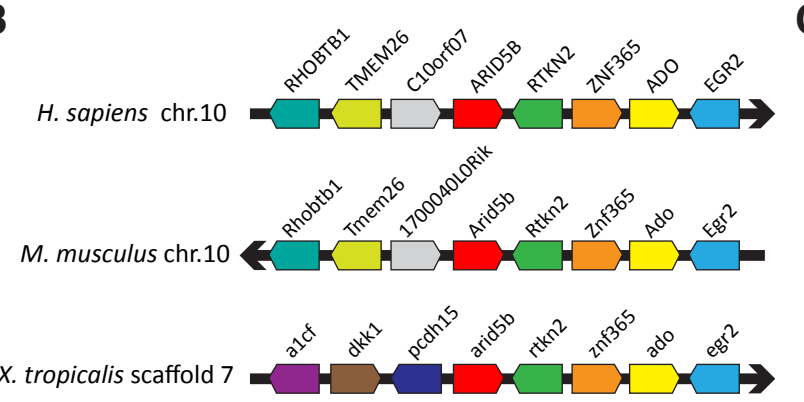

C

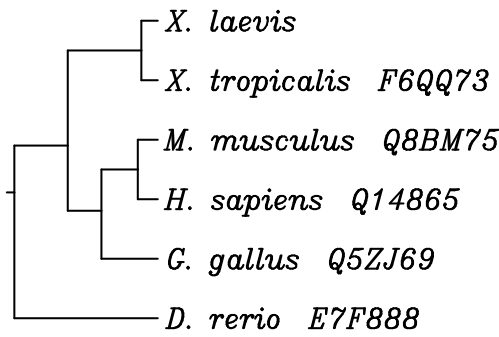

Fig. 1. Characterization of a $X$. laevis ortholog of arid5b. (A) Predicted ARID domain amino acid sequence comparison: G. gallus (ac Q5ZJ69); H. sapiens (ac Q14865); X. tropicalis (F6QQ73); D. rerio (E7F888). (B) Synteny blocks containing arid5b genes in $\mathrm{H}$. sapiens; $\mathrm{M}$. musculus and $\mathrm{X}$. tropicalis genomes. Genes organization in the humanarid5b gene region was used as basis for comparison. Chromosomallocalization is indicated. The scaffold number is given for $\mathrm{X}$. tropicalis. Relative spacing between the genes is not shown. (C) Phylogenetic tree of arid5b genes from various vertebrate species constructed using the neighbour-joining method. Accession numbers used are shown. 
putative coding sequence, and 634 nt of 3'UTR. It is identical to the sequence of the long isoform, in line with human arid5b isoform2 sequence data, which only differs from isoform 1 at the start ATG. Using different sets of primers, we have then tried to amplify the entire short isoform by end-to-end PCR but failed to amplify a full cDNA. Using forward primers corresponding to exon $4 \mathrm{~b}$ sequence, and reverse primers at different levels of the sequence obtained by 3' RACE-PCR, we could only amplify a partial cDNAs encoding the first 476 aa of the short isoform. This cDNA corresponds to exons 4b, 5-9 and part of exon 10 (accession number HG518327). Whether the short isoform is ending at the same stop codon as the long one therefore remains unclear. Nonetheless, a 3'sequence for the short isoform mRNA distinct from that of the long isoform would imply an alternative splicing within exon10, that is not occurring with human arid5b isoform2. Together, these data show that the short isoform 1 lacks the first $243 \mathrm{~N}$-terminal amino acids of the long isoform2 (Fig. S2). Using InterProSCan software and performing an extensive analysis of the litterature on Arid5 family members, we could not find any known domain in this region, precluding to identify any functional difference between the two isoforms.

A

total arid5b (normalized to ODC)

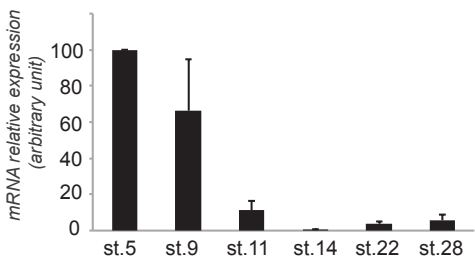

B arid5b isoform 1 (normalized to ODC)

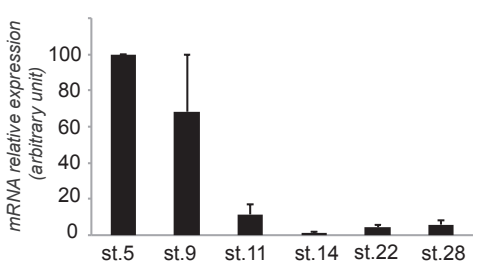

C arid5b isoform2 (normalized to ODC)

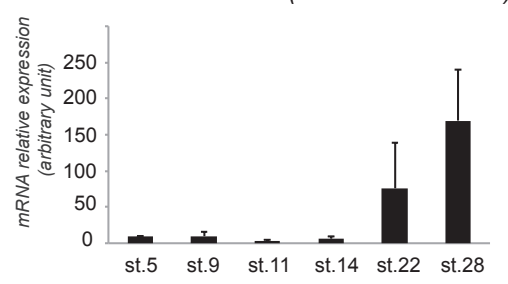

\section{Genomic synteny and molecular phylogeny}

In order to further confirm the identity of the Xenopus ortholog of arid5b, we have examined the synteny maps from human, mouse and $X$. tropicalis genomes. Synteny maps were obtained from Ensembl genome browser (release 74, December 2013) and JGI genome browser (X. tropicalis version 7.1). Synteny maps are shown in Fig.1B. Flanking genes are partially conserved between arid5b genes in human, mouse and $X$. tropicalis genomes. The conserved syntenic region flanks the 3' end of arid5b. It comprises rkn2, znf365, ado and egr2 genes. Genes flanking the 5' end of arid5b in $X$. tropicalis genome totally differ from those flanking human or mouse arid5b. Phylogenetic analysis indicates that Xenopus
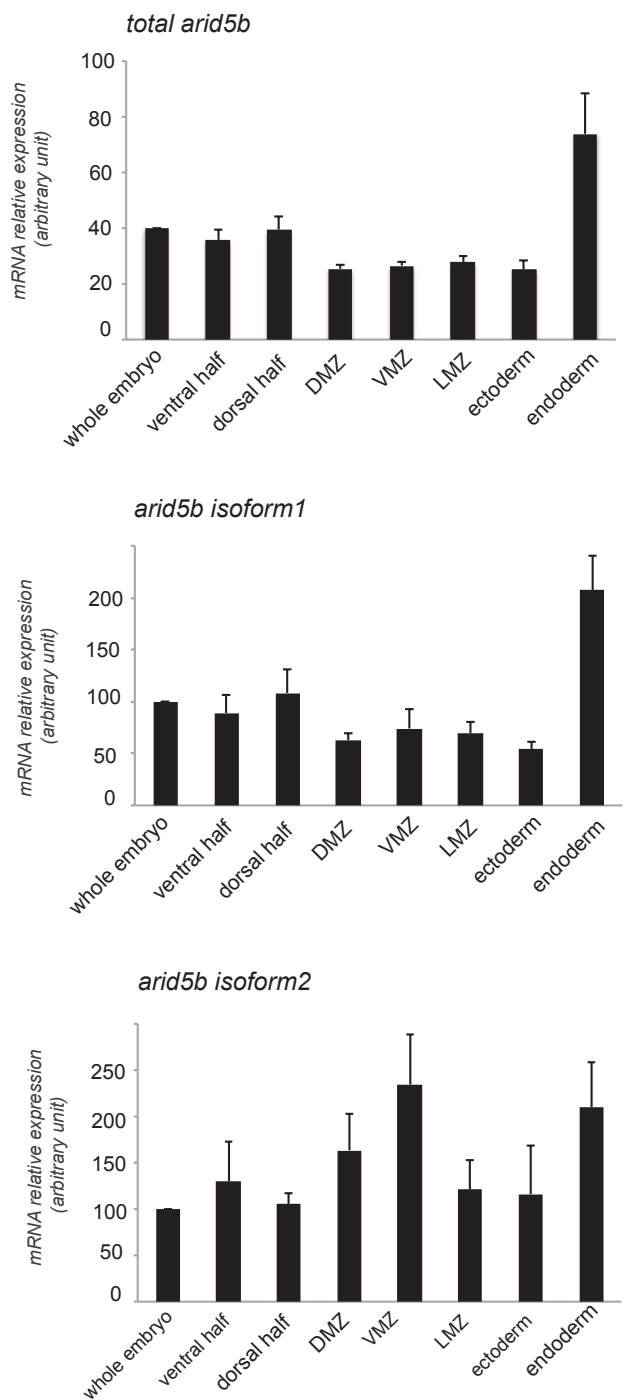

Fig. 2 (Left). Temporal expression of arid5b during Xenopus embryonic development. Expression of arid5b analysed by RT-qPCR at cleavage (stages 5 and 9), gastrula (stage 11), neurula (stages 14) and tailbud stages (stages 22 and 28). Primers were designed in order to amplified either both isoforms (total arid5b) (A), isoform1 (B,D) or isoform2 (C,E). The relative quantities of mRNA were determined with ODC (A,B,C) or total arid5b (D,E) mRNA as the endogenous reporter. Isoform1 is strongly maternally expressed; isoform2 is mainly expressed during tailbud stages. Average values from three independent experiments.

Fig. 3 (Right). Expression of arid5b at the early gastrula stage. RT-qPCR analyses for total arid5b, isoform1 and isoform2 were performed on dissected explants from early gastrula stage embryo (stage 10.5). Embryos were dissected either into ventral and dorsal halves or into dorsal marginal zone (DMZ), ventral marginal zone (VMZ), lateral marginal zone (LMZ) ectoderm and endoderm. Both isoforms are ubiquitously expressed at the early gastrula stage. Average values from three independent experiments. 
arid5b is relatively distant from the zebrafish ortholog. Tetrapod orthologs appear to be more closely related, with the chicken ortholog being the most closely related to Xenopus arid5b (Fig. 1C).

\section{Spatial and temporal expression of arid5b during $\mathrm{X}$. laevis development}

Temporal expression of ari5b during early $X$. laevis development was examined by RT-qPCR. Specific primers were designed in order to amplify either both arid5b isoforms (total arid5b), isoform 1 or isoform2 (Fig. S3). Isoform 1 was strongly expressed during cleavage stages; its expression declined during gastrulation and neurulation, then it increased at tailbud stages (Fig. 2B). In contrast, isoform2 transcripts were scarcely detected at the pretailbud stages, but became detectable during organogenesis and persisted at least up to the late tailbud stage (stage 28) (Fig.
2C). Notably, the temporal profile of isoform 1 is almost identical to the profile obtained for total arid5b, suggesting that isoform 1 is the main isoform to be expressed during embryonic development (Fig.2 A,B). In agreement with this idea, when normalized to total arid5b, relative expression of isoform 1 was constant during embryonic development while relative mRNA expression of isoform2 increased during tailbud stages (Fig.2 D,E). Thus, isoform1 and isoform2are differentially expressed during development. Isoform 1 is strongly expressed maternally and is the main isoform expressed during embryonic development. Isoform2 expression is essentially restricted to tailbud stages.

Next, we studied the spatial expression pattern of arid5b by whole mount in situ hybridization using two probes: the first encompassing nucleotide 775 to 1612 of isoform 1 (which corresponds to nucleotides 46 to 853 of isoform2); the second corresponding to
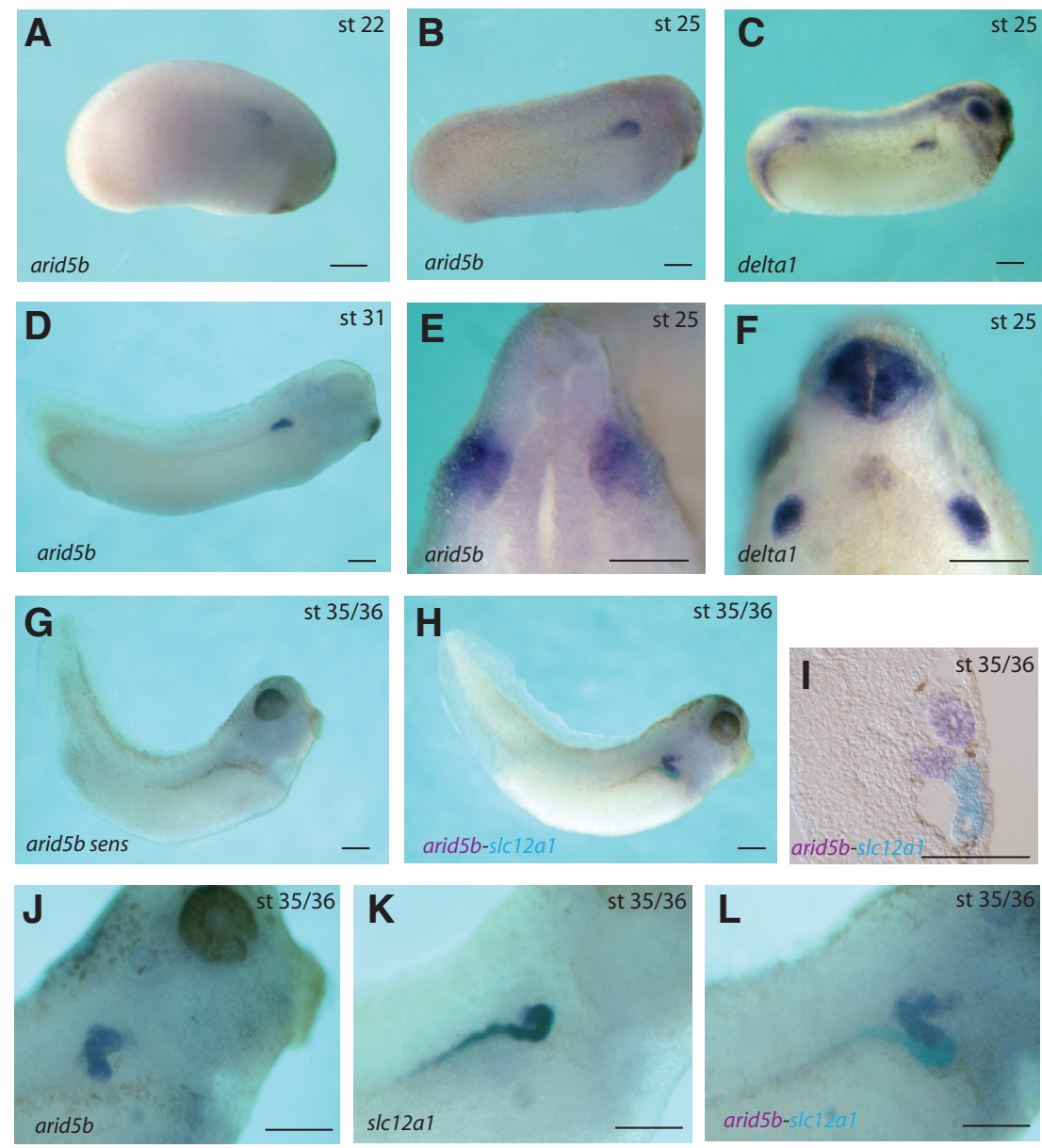

Fig. 4 (Left). Spatial expression of arid5bduring Xenopus development. In situ hybridization of whole $(\mathbf{A}-\mathbf{D}, \mathbf{G}-\mathbf{L})$ or transverse fractured embryos $(\mathbf{E}, \mathbf{F})$ at the indicated stages of development with antisense probe for arid5b (nucleotide 775 to 1612 of isoform1) (A, B, D,E,H,J,L,I),
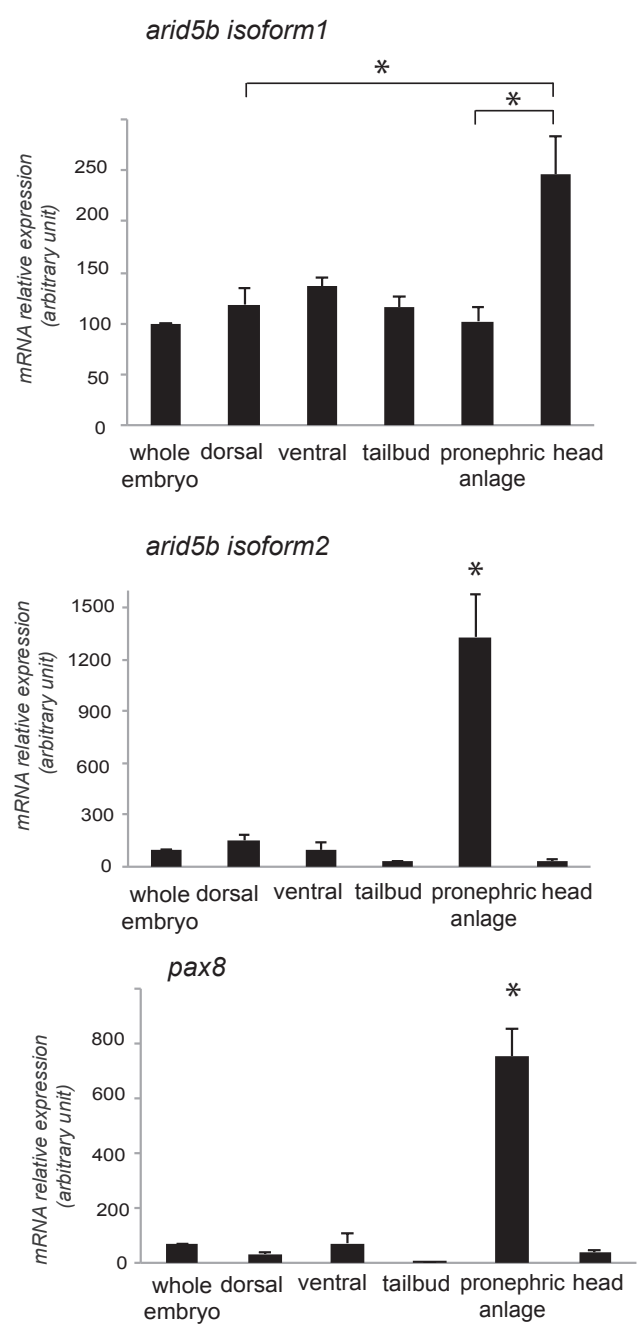

delta1 (C, F), slc12a1 (I, $\mathbf{K}, \mathbf{L})$ and with control sense probe for arid5b (G). Lateral views with anterior to the right $(\mathbf{A}-\mathbf{D}, \mathbf{G}, \mathbf{H}, \mathbf{J}-\mathbf{L})$. Transverse section at the level of the proximal pronephric tubule. In I and L, arid5b is revealed in light blue and slc12a1 in purple. Arid5b mRNAs are detected in the anterior pronephric anlage during tailbud stages in a broader domain than delta1. At tadpole stage 35/36, arid5b expression is restricted to the proximal part of the tubule and does not overlap with slc12a1 expression which is specific for the intermediate and the first segment of the distal tubule. Scale bars are $0.3 \mathrm{~mm}$.

Fig. 5 (Right). Arid5b isoform2 is specifically expressed in the pronephros. Expression of isoform1, isoform 2 and pax8 analysed by $R T-q P C R$ in different embryonic regions (see materials and methods) dissected at tailbud stage 25. Isoform2 as well as pax8 are strongly expressed in pronephric anlage in comparison to other embryonic tissues (statistically significant for all). Isoform1 is slightly more expressed in the head in comparison to other tissues. Average values from three independent experiments. ${ }^{*} \mathrm{P}<0.05$. 
arid5b isoform 1

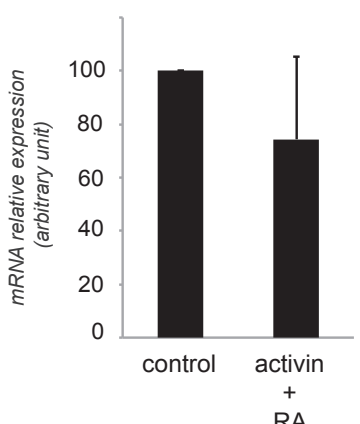

RA

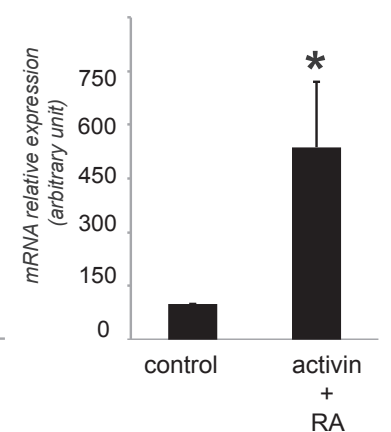

RA

pax8

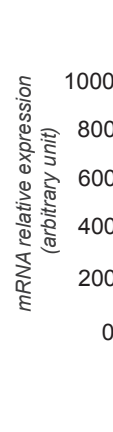

nucleotide 529 to 3564 of isoform1. Both probes gave the same expression pattern (Fig.4 and data not shown). At cleavage, gastrula and neurula stages, embryos were uniformly stained indicating that arid $5 b$ mRNAs were ubiquitously distributed (not shown). RTqPCR analysis of dissected explants from early gastrula confirmed this observation and further showed that none of the two isoforms showed a regionalized expression (Fig. 3). At the early tailbud stage, a specific signal was detected in the pronephric region by in situ hybridization (Fig. 4A). At stage 25, arid5b was strongly expressed in the antero-dorsal part of the pronephric anlage. In comparison with delta1 expression which is restricted to the most antero-dorsal part of the anlage ((Rones et al., 2000) et Fig.4 C,F), arid5b is expressed in a larger domain that extends more ventrally and medially (fig. 4 B,E). Expression of arid5b remained restricted to the anterior part of the developing pronephros at tailbud stages (Fig. 4D). At tadpole stage 35/36, arid5b mRNAs were localized in the proximal part of the pronephric tubule (Fig. $4 \mathrm{H}, \mathrm{J}, \mathrm{I}, \mathrm{L}$ ). Arid5b expression domain is adjoining to that of scl12a1 which marks the intermediate and the first distal segments (Raciti et al., 2008) (Fig. $4 \mathrm{H}, \mathrm{I}, \mathrm{L}$ ). Thus, from the early tailbud stage onward, arid5b expression is restricted to the developing proximal part of the pronephros. Since our in situ hybridization experiments do not allow to distinguish between the two isoforms, we performed RT-qPCR to analyse the expression levels of each isoform in the pronephric anlage in comparison to the whole embryo and to different embryonic regions. The results clearly showed that isoform 2 is more strongly expressed in the pronephric anlage than in the other tissues at tailbud stage (Fig. 5). No such enrichment was observed for isoform 1 although it is slightly more expressed in the head in comparison to other tissues. As expected, the kidney marker pax8 was found to be strongly expressed in the pronephric anlage (Fig. 5). It has been previously shown that treatment of blastula animal cap ectoderm with activin $A$ and retinoic acid (RA) results in the formation of pronephric tubules at high frequency (Ariizumi and Asashima, 2001). We studied whether this treatment could upregulate arid5b isoforms expression. We showed that isoform 2 but not isoform 1 expression is upregulated in response to activin $\mathrm{A}$ and RA (Fig. 6). This result is in agreement with a specific pronephric signature of the isoform2.

In summary, we have cloned the $X$. laevis ortholog of arid5b, identified two isoforms and examined their expression pattern during embryonic development. The two isoforms are differentially expressed: isoform 1 is strongly expressed maternally, while isoform 2 is specifically expressed in the pronephric anlage at tailbud

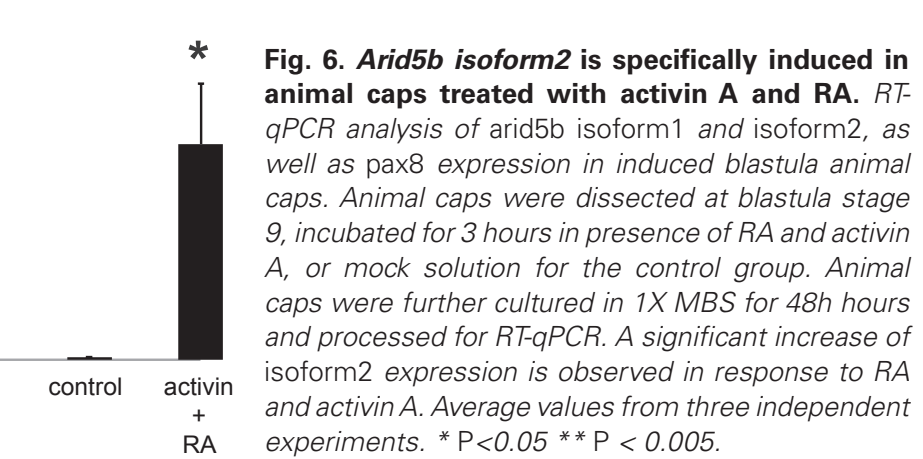

stage. These results indicate that the two arid5b isoforms likely play different roles during early Xenopus development.

\section{Materials and Methods}

\section{Molecular cloning and bioinformatic analyses}

The partial IMAGE clone 696,6480 was obtained from RZPD ImaGenes. RACE-PCR was performed with the SMARTer ${ }^{\mathrm{TM}}$ RACE cDNA amplification kit (Clontech). End-to-end PCR was carried out according to manufacturer instructions with Advantage 2 polymerase (Clontech), and stage 35/36 embryo cDNA prepared as described (Le Bouffant et al., 2012). Amino acid sequence comparison were performed with MultAlin software (http://multalin. toulouse.inra.fr/multalin/) (Corpet, 1988) and CLUSTAL W (version 1.83).

\section{Embryos, explants dissection, animal cap assay}

Xenopus lævis were purchased from the CNRS Xenopus breeding Center (Rennes, France). Embryos were obtained after artificial fertilization, and were raised in modified Barth's solution (MBS). Stages were according to the normal table of Xenopus lævis (Nieuwkoop and Faber, 1967). Dissections were all performed in $1 \mathrm{X}$ MBS on $1 \%$ agar-coated dishes. Presumptive ectoderms (animal cap) were isolated from blastula stage embryos (stages 8-9) and immediately transferred into 1X MBS, $0.1 \%$ BSA in the presence of recombinant human activin A (10 ng/mL, Sigma) and all-trans retinoic acid $\left(10^{-4} \mathrm{M}\right.$, Sigma) or DMSO alone (1/250). The animal caps were incubated for $3 \mathrm{hrs}$, after which they were washed twice in 1X MBS and further cultured for $48 \mathrm{hrs}$. Explants comprising the pronephric anlage were dissected from early tailbud embryos (stage 21). Using platinum loop and wire, stage 25 embryos were dissected into several pieces: the head, the tail, the truncal dorsal part (essentially somites, neural tube and notochord) and the truncal ventral part (mainly endoderm, ventral and lateral mesoderm). Pronephric anlagen were isolated from somitic and lateral plate mesoderm and separated from the underlying endoderm. The overlying ectoderm was kept. Explants were immediately processed for RT-qPCR.

\section{Real-time quantitative PCR}

RT-qPCR analyses were carried out as previously reported in (Le Bouffant et al., 2012). Sequences of oligonucleotides used are the following: arid5b: forward: 5'TATGTTTCAAGCTGCGCAAAA3', reverse: 5'CCATTGCCTCCGTGCAGTA3'; arid5b isoform 1 forward: 5'CCCAGAAGATACCCCCAAGG3', reverse:5'ACTTCATGCTCTCCGTGGCT3'; arid5b isoform2: forward: 5'TGCTCTGTGGCGTTCATGAG3'

reverse: 5'TCGACTAGCATCTGTCTCGTTTGT3'; pax8: forward: 5'CAGCAATTTCAATATAGGTCACGG3', reverse 5'TCCATTCACAAAAGCCCCAC3'; ODC: forward:5'GGGCAAAGGAGCTTAATGTGG3', reverse 5'TGCCAACATGGAAACTCACAC3'. The Comparative Ct method was used to determine the relative quantities of mRNA, using ODC mRNA as the endogenous reporter except for figure 2D,E for which arid5b was used. Same results were obtained using $\beta$-actin mRNA as the endogenous 
reporter instead of $O D C$ (data not shown). Each RNA sample was analysed in duplicate. Each data point represents the mean \pm SEM of at least three independent experiments. Data were analysed using $\mathrm{R}$ Commander $(\mathrm{R}$ software) by paired Student's t-test.

\section{In situ hybridization}

Whole mount in situ hybridization for arid5b, delta1 (Rones et al., 2000), and slc12a1 (Raciti et al., 2008) were carried out as previously reported (Cartry et al., 2006). The antisense and control sense RNA probes for arid5b were generated from linearized plasmids containing cDNA sequences from nucleotide 529 to 3564 of isoform 1 and from nucleotide 775 to 1612 of isoform 1 (which corresponds to nucleotides 46 to 853 of isoform2). The arid5b RNA probes were subjected to limited alkaline hydrolysis in two volumes of carbonate buffer ( $\left.60 \mathrm{mM} \mathrm{Na}_{2} \mathrm{CO}_{3}, 40 \mathrm{mM} \mathrm{NaHCO}_{3}, \mathrm{pH} 10.2\right)$ for $5 \mathrm{~min}$ at $60^{\circ} \mathrm{C}$ to reduce its size and increase its access to tissues. The hydrolysis was terminated by adding an equal volume of neutralizing solution (1 M Tris- $\mathrm{HCl}$, pH 8.0, containing $1.5 \mathrm{M} \mathrm{NaCl}$ ). Hydrolyzed fragments were precipitated with ethanol.

\section{Acknowledgements}

We thank S. Autier and E. Manzoni for animal care, E.A. Jones and E. Bellefroid for plasmids. This work was supported by grants from CNRS and from University' Pierre et Marie Curie. We acknowledge funding from Emergence-UPMC-2009 research program.

\section{References}

ARIIZUMI, T. and ASASHIMA, M. (2001). In vitro induction systems for analyses of amphibian organogenesis and body patterning. Int J Dev Biol 45: 273-279.

BABA, A., OHTAKE, F., OKUNO, Y., YOKOTA, K., OKADA, M., IMAI, Y., NI, M., MEYER, C.A., IGARASHI, K., KANNO, J. et al., (2011). PKA-dependent regulation of the histone lysine demethylase complex PHF2-ARID5B. Nat Cell Biol 13: 668-75.

CARTRY, J., NICHANE, M., RIBES, V., COLAS, A., RIOU, J.F., PIELER, T., DOLLE, P., BELLEFROID, E.J. and UMBHAUER, M. (2006). Retinoic acid signalling is required for specification of pronephric cell fate. Dev. Biol. 299: 35-51.

COLLINS, R.T., FURUKAWA, T., TANESE, N. and TREISMAN, J.E. (1999). Osa associates with the Brahma chromatin remodeling complex and promotes the activation of some target genes. EMBO J 18: 7029-7040.

CORPET, F. (1988). Multiple Sequence Alignment with Hierarchical-Clustering. Nucleic Acids Res 16: 10881-10890.

KOZMIK, Z., MACHON, O., KRALOVA, J., KRESLOVA, J., PACES, J. and VLCEK, C. (2001). Characterization of mammalian orthologues of the Drosophila osa gene: cDNA cloning, expression, chromosomal localization, and direct physical interaction with Brahma chromatin-remodeling complex. Genomics 73: 140-148.
LAHOUD, M.H., RISTEVSKI, S., VENTER, D.J., JERMIIN, L.S., BERTONCELLO, I., ZAVARSEK, S., HASTHORPE, S., DRAGO, J., DE KRETSER, D., HERTZOG, P.J. et al., (2001). Gene targeting of Desrt, a novel ARID class DNA-binding protein, causes growth retardation and abnormal development of reproductive organs. Genome Res 11: 1327-1334.

LE BOUFFANT, R., WANG, J.H., FUTEL, M., BUISSON, I., UMBHAUER, M. and RIOU, J.F. (2012). Retinoic acid-dependent control of MAP kinase phosphatase-3 is necessary for early kidney development in Xenopus. Biol Cell 104: 516-532.

LUBON, H., PITTIUS, C.W. and HENNIGHAUSEN, L. (1989). Invitro Transcription of the Mouse Whey Acidic Protein Promoter Is Affected by Upstream Sequences. FEBS Letters 251: 173-176.

NIEUWKOOP, P.D. and FABER, J. (1967). Normal table of Xenopus lævis (Daudin). North-Holland Publishing Company, Amsterdam.

PATSIALOU, A., WILSKER, D. and MORAN, E. (2005). DNA-binding properties of ARID family proteins. Nucleic Acids Res 33: 66-80.

RACITI, D., REGGIANI, L., GEFFERS, L., JIANG, Q., BACCHION, F., SUBRIZI, A.E., CLEMENTS, D., TINDAL, C., DAVIDSON, D.R., KAISSLING, B. et al., (2008). Organization of the pronephric kidney revealed by large-scale gene expression mapping. Genome Biol 9: R84.

RISTEVSKI, S., TAM, P.P.L., KOLA, I. and HERTZOG, P. (2001). Desrt, an AT-rich interaction domain family transcription factor gene, is an early marker for nephrogenic mesoderm and is expressed dynamically during mouse limb development. Mech. Dev. 104: 139-142.

RONES, M.S., MCLAUGHLIN, K.A., RAFFIN, M. and MERCOLA, M. (2000). Serrate and Notch specify cell fates in the heart field by suppressing cardiomyogenesis. Development 127: 3865-3876.

SCHMAHL, J., RAYMOND, C.S. and SORIANO, P. (2007). PDGF signaling specificity is mediated through multiple immediate early genes. Nature Genetics 39: 52-60.

WATANABE, M., LAYNE, M.D., HSIEH, C.M., MAEMURA, K., GRAY, S., LEE, M.E. and JAIN, M.K. (2002). Regulation of smooth muscle cell differentiation by ATrich interaction domain transcription factors Mrf2 alpha and Mrf2 beta. Circ Res 91: 382-389.

WHITSON, R.H., HUANG, T. and ITAKURA, K. (1999). The novel Mrf-2 DNA-binding domain recognizes a five-base core sequence through major and minor-groove contacts. Biochem Biophys Res Commun 258: 326-331.

WHITSON, R.H., TSARK, W., HUANG, T.H. and ITAKURA, K. (2003). Neonatal mortality and leanness in mice lacking the ARID transcription factor Mrf-2. Biochem Biophys Res Commun 312: 997-1004.

YAMAKAWA, T., SUGIMOTO, K., WHITSON, R.H. and ITAKURA, K. (2010). Modulator recognition factor-2 regulates triglyceride metabolism in adipocytes. Biochem Biophys Res Commun 391: 277-281.

YAMAKAWA, T., WHITSON, R.H., LI, S.L. and ITAKURA, K. (2008). Modulator recognition factor-2 is required for adipogenesis in mouse embryo fibroblasts and 3T3-L1 cells. Molec. Endocrinol. 22: 441-453. 


\section{Further Related Reading, published previously in the Int. J. Dev. Biol.}

Involvement of the eukaryotic initiation factor 6 and kermit2/gipc2 in Xenopus laevis pronephros formation Margherita Tussellino, Nadia De Marco, Chiara Campanella and Rosa Carotenuto

Int. J. Dev. Biol. (2012) 56: 357-362

http://dx.doi.org/10.1387/ijdb.120009nd

\section{Developmental expression of Xenopus short-chain dehydrogenase/reductase 3}

Richard K.T. Kam, Yonglong Chen, Sun-On Chan, Wood-Yee Chan, Igor B. Dawid and Hui Zhao Int. J. Dev. Biol. (2010) 54: 1355-1360

http://dx.doi.org/10.1387/ijdb.092984rk

Competition for ligands between FGFR1 and FGFR4 regulates Xenopus neural development Masahiro Yamagishi and Harumasa Okamoto

Int. J. Dev. Biol. (2010) 54: 93-104

http://dx.doi.org/10.1387/ijdb.092849my

Lef1 plays a role in patterning the mesoderm and ectoderm in Xenopus tropicalis Giulietta Roël, Yoony Y.J. Gent, Josi Peterson-Maduro, Fons J. Verbeek and Olivier Destrée Int. J. Dev. Biol. (2009) 53: 81-89

http://dx.doi.org/10.1387/ijdb.072395gr

Expression of complement components coincides with early patterning and organogenesis in Xenopus laevis

Valérie A. McLin, Cheng-Hui Hu, Rina Shah and Milan Jamrich

Int. J. Dev. Biol. (2008) 52: 1123-1133

http://dx.doi.org/10.1387/ijdb.072465v

Temporal and spatial expression patterns of FoxN genes in Xenopus laevis embryos Maximilian Schuff, Antje Rössner, Cornelia Donow and Walter Knöchel

Int. J. Dev. Biol. (2006) 50: 429-434

http://dx.doi.org/10.1387/ijdb.052126ms

The zic1 gene is an activator of Wnt signaling

Christa S. Merzdorf and Hazel L. Sive

Int. J. Dev. Biol. (2006) 50: 611-617

http://dx.doi.org/10.1387/ijdb.052110cm

The role of XTRAP-gamma in Xenopus pronephros development

Dong-hui Li, Techuan Chan, Reiko Satow, Shinji Komazaki, Kouhei Hashizume and Makoto Asashima

Int. J. Dev. Biol. (2005) 49: 401-408 http://dx.doi.org/10.1387/ijdb.052005dl

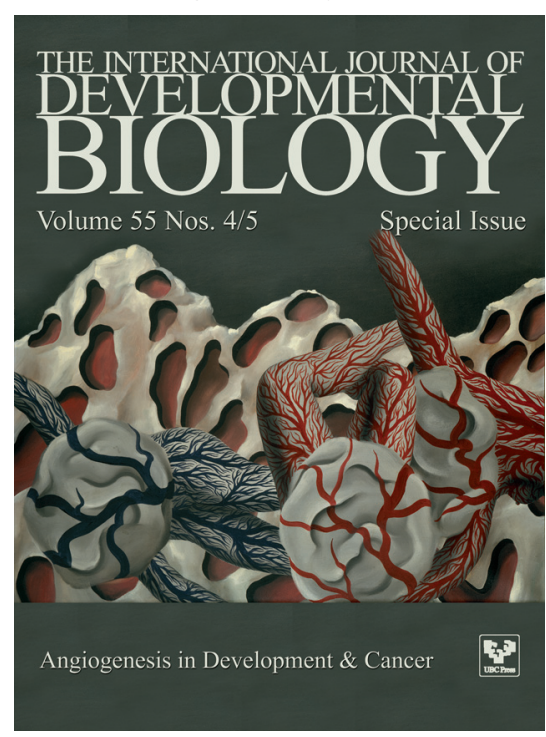

5 yr ISI Impact Factor $(2011)=2.959$
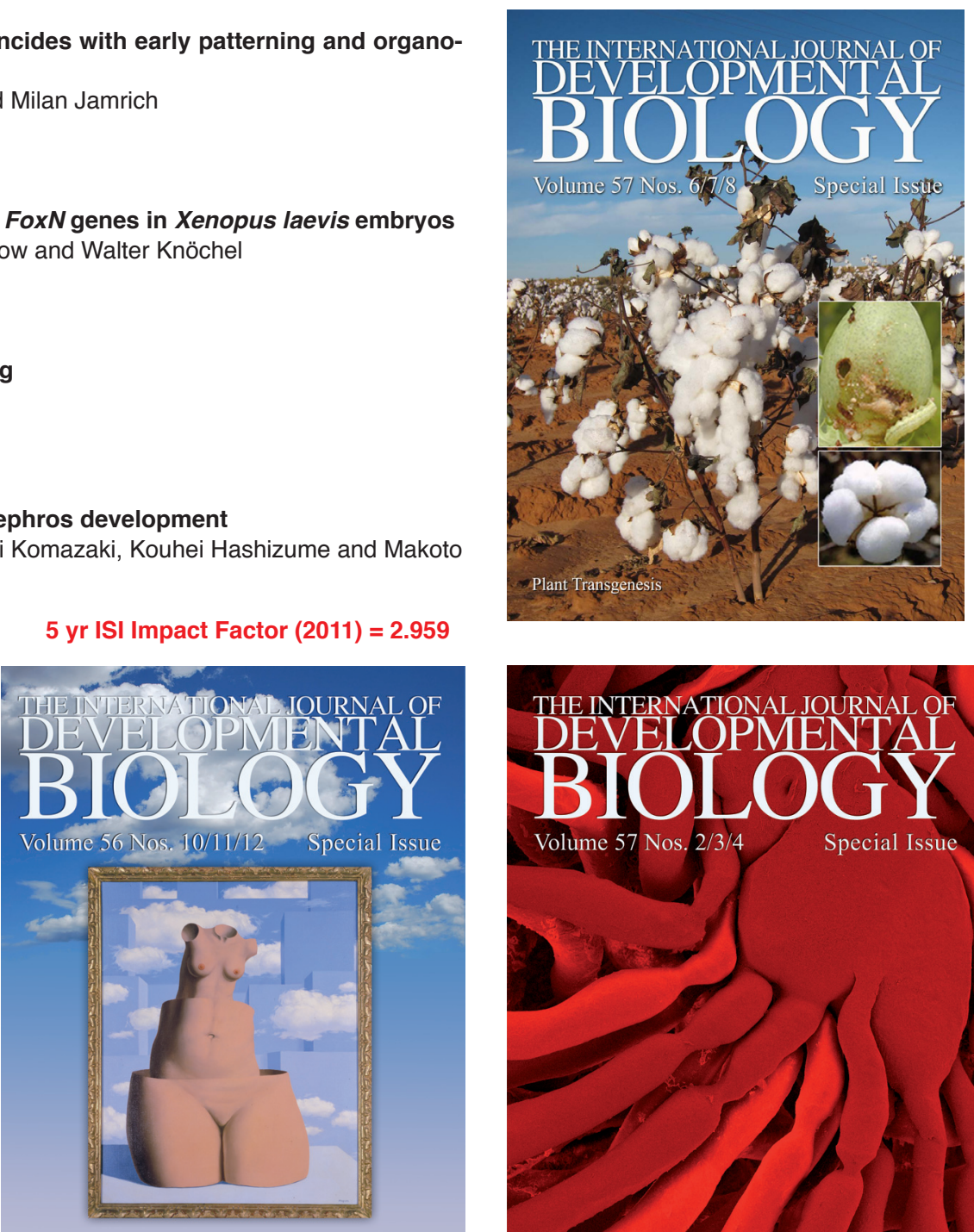

Female Germ Cells in Development \& Tumors

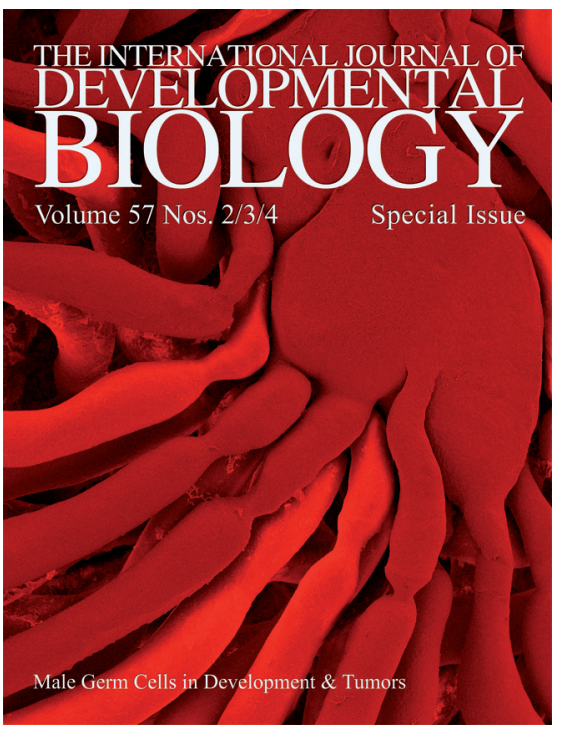

